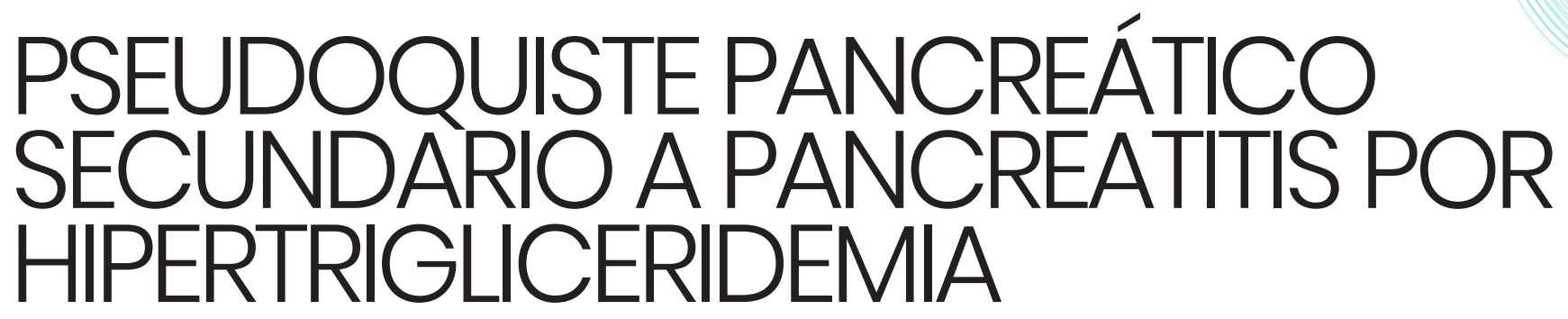

\title{
PANCREATIC PSEUDOCYST SECONDARY TO HYPERTRIGLYCERIDEMIA PANCREATITIS
}

\section{Abaca DA, Arcos JA}

Sanatorio Juan XXIII. General Roca. Río Negro, Argentina.

\section{Resumen}

Los pseudoquistes pancreáticos son las lesiones quísticas pancreáticas más frecuentes. Se originan como consecuencia de una pancreatitis aguda (10-20\%), una pancreatitis crónica (20$40 \%$ ), postraumatismo (5-10\%) o postoperatoria. Tras uno de estos episodios se forman colecciones líquidas, la mayoría de ellas resuelven espontáneamente, de lo contrario a medida que transcurren las semanas terminan siendo encapsuladas por el tejido inflamatorio formando un quiste sin pared epitelial. Independientemente de su tamaño tienen indicación terapéutica solo aquellos complicados o sintomáticos. El tratamiento inicial de elección es el endoscópico, reservando la cirugía para aquellos pacientes que han fallado estos procedimientos.

Presentamos un caso de pseudoquiste pancreático por pancreatitis secundaria a hipertrigliceridemia. Esta es la tercera causa más frecuente de pancreatitis detrás de la biliar y el alcohol, con una incidencia del 1-5\%.

\section{CORRESPONDENCIA}

Dario abaca

Sanatorio Juan XXIII

1468 General Roca. Río Negro. Argentina.

dario.abaca.a@gmail.com

Fecha de envío: 07/05/2020

Fecha de aceptación: 18/06/2020
Palabras clave: pseudoquiste pancreático, pancreatitis secundaria a hipertrigliceridemia, tratamiento endoscópico.

\section{Abstract}

Pancreatic pseudocysts are the most frequent pancreatic cystic lesions. They originate as a consequence of acute pancreatitis (10-20\%), chronic pancreatitis $(20-40 \%)$, post-trauma $(5-10 \%)$ or postoperative. After one of these episodes liquid collections are formed, the most of them resolve spontaneously, otherwise as the weeks pass they end up being encapsulated by the inflammatory tissue forming a cyst without epithelial wall. Independently of their size, only those that are complicated or symptomatic have a therapeutic indication. The initial treatment of choice is endoscopic, reserving surgery for those patients who have failed these procedures. We present a case of pancreatic pseudocyst due to pancreatitis secondary to hypertriglyceridemia. This is the third most frequent cause of pancreatitis behind the biliary and alcohol, with an incidence of $1-5 \%$

Keywords: pancreatic pseudocyst, pancreatitis secondary to hypertriglyceridemia, endoscopic treatmen. 


\section{Introducción}

Los pseudoquistes pancreáticos son colecciones liquidas encapsuladas por tejido inflamatorio no epitelizada, que maduran al cabo de 2 a 6 semanas. Se presentan como complicación de una pancreatitis aguda, crónica, postraumatismo o postoperatoria. La mayoría resuelven espontáneamente, siendo indicación de tratamiento solo aquellos complicados o sintomáticos. Dentro de sus complicaciones tenemos; la hemorragia, la infección, hipertensión portal, obstrucción de la vía biliar y ruptura. El drenaje endoscópico se ha convertido en la primera opción terapéutica.

Presentamos un caso de pseudoquiste pancreático por pancreatitis secundaria a hipertrigliceridemia con factores asociados que resolvió con tratamiento médico y drenaje endoscópico.

\section{Caso clínico}

Paciente de 37 años, con antecedentes de hipotiroidismo no controlado y ex-tabaquista. No refiere conocerse diabético pero ingreso con glucemias elevadas. Comenzó con dolor súbito e intenso en epigastrio acompañado de vómitos. Al examen físico piel y mucosa secas, afebril, taquicárdico y taquipneico. Presentaba xantomas en las superficies extensoras de los brazos y piernas. Abdomen doloroso a la palpación en epigastrio sin signos de irritación peritoneal. Se solicita laboratorio, ecografía y tomografía computada. En el laboratorio se puede observar suero de aspecto lipémico; glóbulos blancos: 8300, glucemia 2,49; amilasa 424; GOT 22, GPT 45; FAL 125; triglicéridos: 2700. Ecografía: leve esteatosis hepática, vesícula alitiásica, vía biliar no dilatada. En la tomografía se puede observar proceso inflamatorio pancreático en evolución (Baltazar C) (Figura 1). Con diagnóstico de pancreatitis aguda por hipertrigliceridemia se decide su internación. Se inicia tratamiento con analgesia, hidratación endovenosa, ayuno e infusión de insulina. A las 48 horas de iniciado el tratamiento se observa descenso significativo de triglicéridos (Figura 2). Al cabo de 3 semanas el paciente comienza con fiebre, intolerancia oral y dolor en epigastrio. Se realiza tomografía que evidencia desdibujo pancreático y colección retrogástrica (Figura 3). Con diagnóstico presuntivo de pseudoquiste pancreático infectado se decide realizar drenaje endoscópico. En quirófano con anestesia total, se realiza endoscopia digestiva alta, se evidenció abombamiento en la cara posterior del antro gástrico. Se realiza punción de la pared hasta penetrar en la cavidad del pseudoquiste, extrayendo material purulento (Figura 4). Se aborda el pseudoquiste con cistotomo, logrando avanzar la guía hidrofílica de 0,035 . Se realiza el drenaje de aproximadamente 5 litros de material purulento (Figura 5). Se retira cistotomo para realizar dilatación con balón hidrostático hasta $10 \mathrm{~mm}$.

Al $4^{\circ}$ día se realiza control endoscópico, observando permeabilidad cistogástrica y adecuada evacuación del pseudoquiste.

Paciente con buena tolerancia al procedimiento, se realiza laboratorio y tomografía de control postoperatorio. Laboratorio con marcado descenso de los valores, en la tomografía se evidencia adecuada evacuación del pseudoquiste.

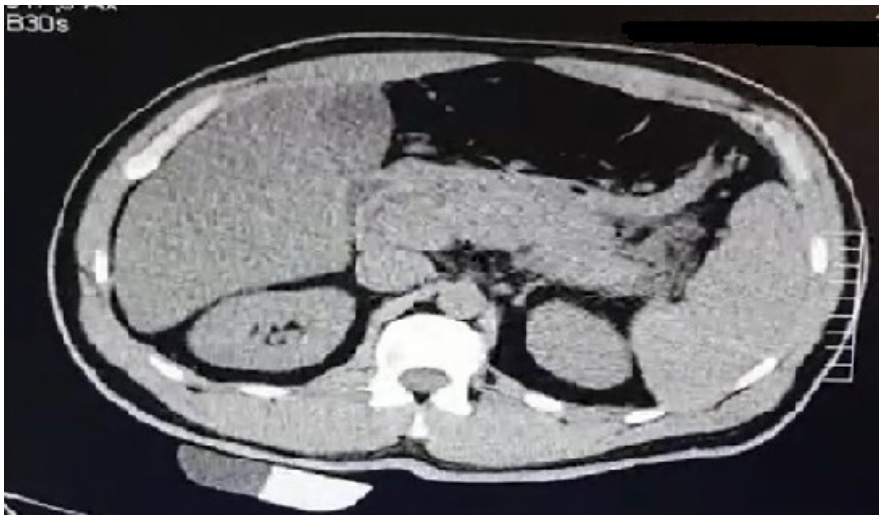

Figura 1

Tomografía computada. Proceso inflamatorio pancreático (Baltazar C).

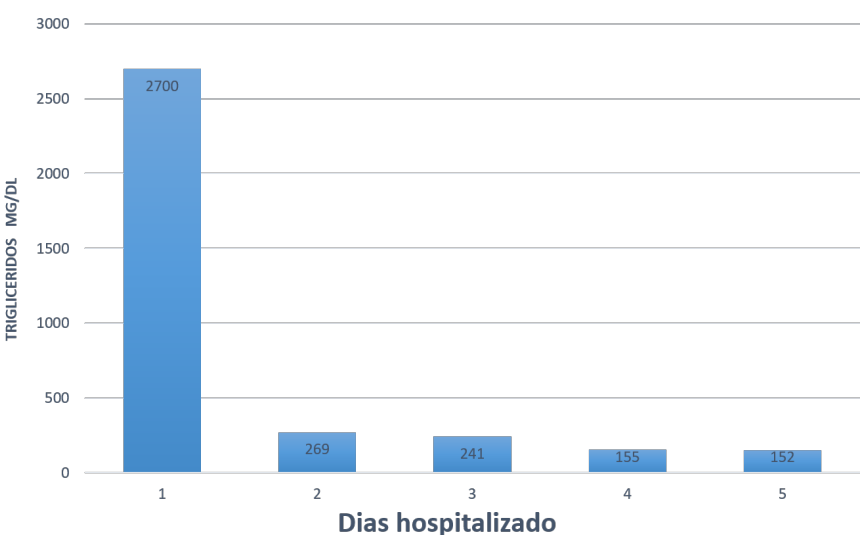

Figura 2

Curva de triglicéridos.

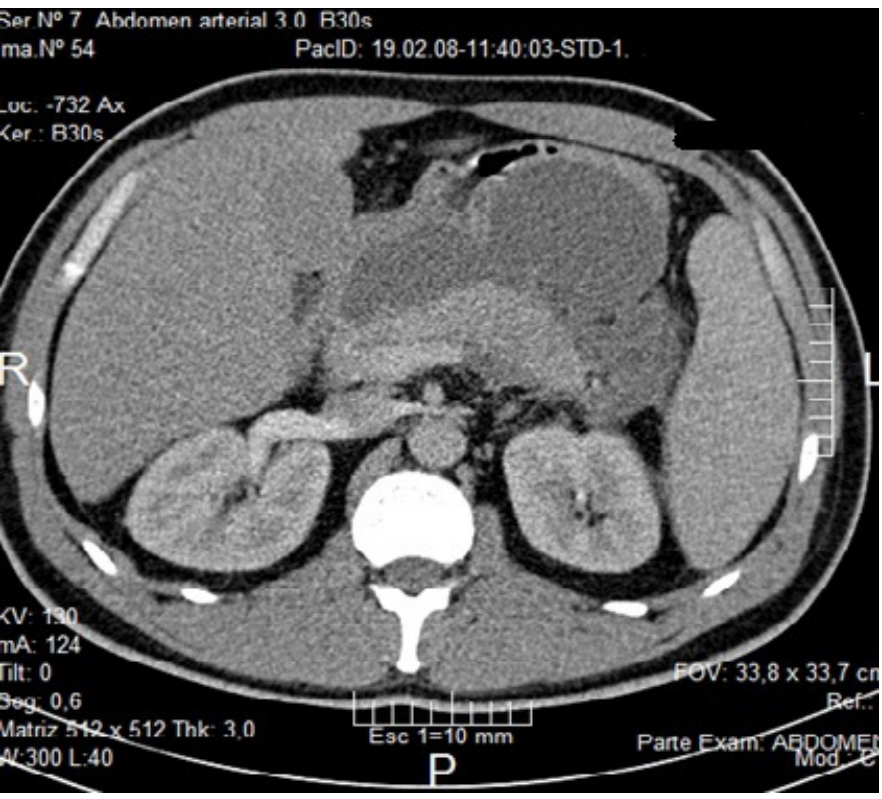

Figura 3

Tomografía computada. Pseudoquiste pancreático retrogástrico. 


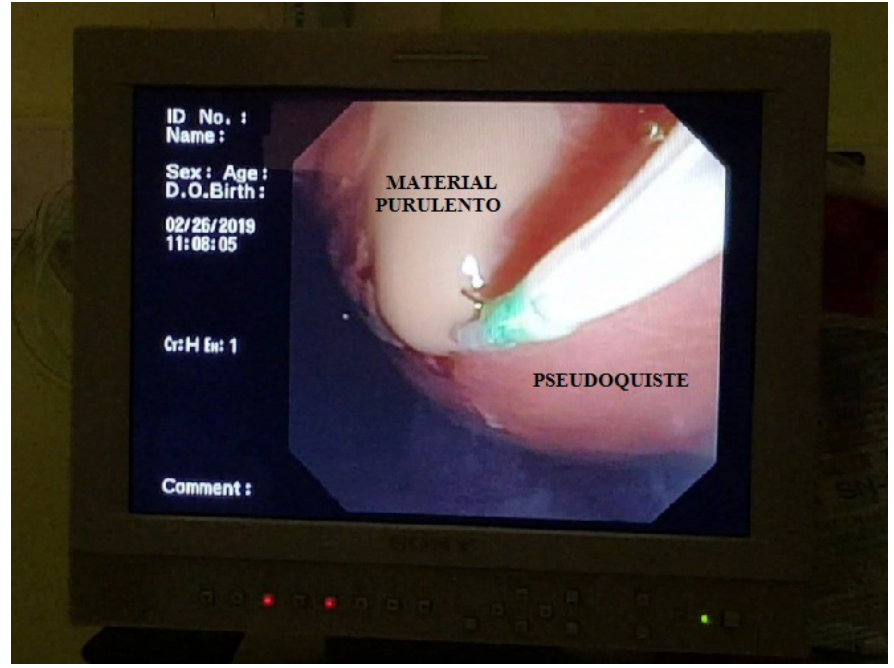

Figura 4

Endoscopia alta. Punción de pseudoquiste infectado abombando en antro gástrico.

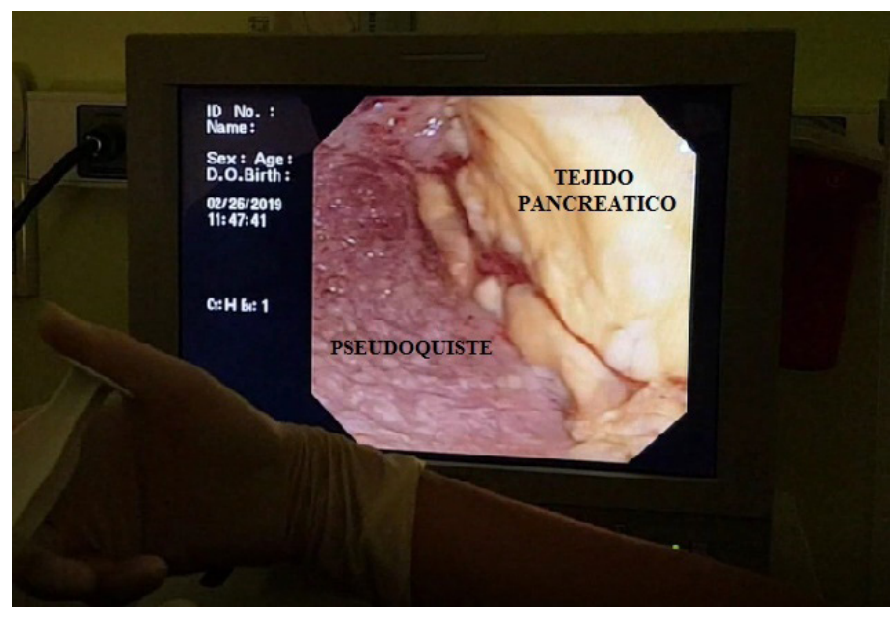

Figura 5

Endoscopia alta. Cavidad de pseudoquiste.

Luego de culminar tratamiento endovenoso con imipenem se decide externación con control de laboratorio y ecografía a la semana. A la fecha no presentó complicaciones ni recurrencia del pseudoquiste. Continúa en tratamiento con hipolipemiantes y dieta, en seguimiento con nutrición y endocrinología.

\section{Discusión}

Los pseudoquistes pancreáticos son colecciones líquidas ricas en amilasa y enzimas pancreáticas que al transcurrir las semanas terminan siendo encapsuladas por el tejido inflamatorio formando un quiste sin pared epitelial. Suelen desencadenarse como consecuencia de una pancreatitis aguda (10-20\%), una pancreatitis crónica (20-40\%), postraumatismo pancreático (5-10\%) o postoperatoria. La hipertrigliceridemia es una causa bien establecida pero subdiagnosticada de pancreatitis aguda, llegando a ser la tercer causa más frecuente detrás de la biliar y el alcohol.
Se presenta con una incidencia entre el 1-5\%. Si bien la pancreatitis por hipertrigliceridemia se presenta con niveles de triglicéridos superiores a $1.000 \mathrm{mg} / \mathrm{dl}$, se han presentado casos con niveles más bajos, aunque es improbable que ocurra con niveles inferiores a $500 \mathrm{mg} / \mathrm{dl}$.

Su etiología puede ser primaria o secundaria. Las primarias son más frecuentes y se asocian a trastornos genéticos en el metabolismo de los lípidos, en cuanto a las causas secundarias pueden ser por obesidad, alcoholismo, hipotiroidismo, diabetes no controlada e inducida por fármacos $\cos ^{1,2,3,4}$.

Diversas teorías explican los mecanismos de acción, un primer mecanismo explica la toxicidad de los ácidos grasos que no se hayan unidos a la albúmina, desencadenando la actividad enzimática pancreática en el tejido acinar. Otro mecanismo de acción se da por la obstrucción pancreática distal por los quilomicrones ocasionando una isquemia que activa una respuesta inflamatoria, por ende mayor actividad de las enzimas pancreáticas. Otro mecanismo atribuible a una alteración genética de la lipoproteinlipasa. Existen mecanismos no conocidos como en el caso del hipotiroidismo que se asocian a una alteración del metabolismo de las lipoproteínas y de los receptores. Se han reportado casos de asociación entre hipotiroidismo y la hipertrigliceridemia ${ }^{5,6}$.

En la mayoría de los casos se arriba al diagnóstico tras estudios negativos que descartan las causas más frecuentes, una característica esencial que puede ayudar a sospechar el diagnóstico de forma precoz es el aspecto lipémico del suero.

Por un lado tenemos el tratamiento de la pancreatitis, que requiere ayuno, hidratación endovenosa y analgesia y por otro el tratamiento de la hipertrigliceridemia. El ayuno tiene un rol fundamental pues favorece el metabolismo de los quilomicrones y de los triglicéridos. Se describe el uso de plasmaféresis, insulina y heparina, siendo estas dos últimas terapéuticas las que mayores descripciones tienen por su fácil disponibilidad.

El empleo de la plasmaféresis no solo permite la disminución de los triglicéridos sino que también realiza una eliminación de citoquinas proinflamatorias permitiendo la perfusión del páncreas. Es un procedimiento eficaz pero invasivo por lo que presenta una serie de complicaciones como fenómenos alérgicos, náuseas, vómitos, dolor torácico, trombosis venosa aguda y tromboembolia pulmonar. Cuando está se encuentra contraindicada, no disponible o el paciente presenta niveles de glucemia mayores a $500 \mathrm{mg} / \mathrm{dl}$ se puede realizar infusión de insulina, esto es posible ya que la mayoría de las hipertrigliceridemias tiene como factor asociado glucemias elevadas.

El uso de la heparina de bajo peso molecular genera controversia, si bien aumenta el nivel de la lipoproteinlipasa rápidamente es metaboliza en el hígado por lo que su uso es relativo.

Algunas series recomiendan el uso de hipolipemiantes, restricción de grasas y la introducción de ácidos grasos omega3, esta medida ayuda a prevenir recurrencias de pancreatitis y prevenir otras complicaciones ${ }^{7,8,9}$. 
Encuanto al pseudoquistes es la lesión quística más frecuente del páncreas. Tiene mayor prevalencia en el sexo masculino, entre la cuarta y quinta década. Se estima que el periodo de maduración de los mismos es de 2 a 6 semanas aproximadamente ${ }^{10,11}$. La mayoría resuelve espontáneamente, si bien el tamaño es el factor más influyente (los < de $5 \mathrm{~cm}$ presentan menor índice de complicaciones y mayor probabilidad de resolución espontánea), se puede destacar otros factores asociados a baja resolución como; localización extrapancreática, presencia de múltiples quistes, localización en cola pancreática, ausencia de comunicación con conducto pancreático principal y grosor de la pared ${ }^{12}$. Generalmente se presentan como únicos, sin embargo cuando hay asociación con el alcohol son múltiples. Su tamaño es variable, pudiendo llegar a medir hasta $30 \mathrm{~cm}$. En cuanto a su localización pueden ser extrapancreáticos o intrapancreaticos (se presentan preferentemente en cuerpo y cola) ${ }^{13}$. Su presentación clínica puede variar, desde pacientes asintomáticos o presentando algún síntoma como dolor abdominal, pérdida de peso, saciedad temprana, náuseas, vómitos, ictericia y fiebre.

Los avances tecnológicos nos permiten realizar un seguimiento de la evolución clínica y hasta guiar conductas terapéuticas. Dentro de los métodos tenemos la ecografía, la tomografía computada, la resonancia magnética, y colangiopancreatografía retrógrada endoscópica (ERCP). La ecografía es el método menos invasivo, no requiere irradiación y nos permite realizar punciones guiadas. Generalmente la tomografía es el método de elección, nos ayuda a diferenciar las colecciones de los pseudoquistes verdaderos y al igual que la ecografía se puede utilizar en las punciones. La colangioresonancia nos permite analizar las relaciones del pseudoquiste con los conductos pancreáticos. La ERCP es un método invasivo que nos permite opacificar los conductos biliares y el conducto de Wirsung estableciendo si existe comunicación con estos.

El tratamiento del pseudoquiste ha cambiado y continúa evolucionando. Previamente al año 2013 se recomendaba el drenaje de lesiones mayores a $6 \mathrm{~cm}$ o si persistían más allá de 6 semanas, pero en dicho año el colegio americano de gastroenterología mediante las guías de manejo de la pancreatitis aguda estableció que los pseudoquistes asintomáticos pueden manejarse de forma conservadora independientemente del tamaño, localización o extensión a estructuras vecinas, siendo sólo pasible de tratamiento aquellos complicados o sintomáticos ya sea por lesión o extensión ${ }^{14}$. El drenaje del pseudoquiste se lo puede dividir en drenaje interno, drenaje externo y la resección. Lo que difiere es el tipo de abordaje, siendo este mismo quirúrgico, endoscópico o percutáneo. Tradicionalmente el abordaje quirúrgico y sobre todo el laparotómico era considerado el gold standard pero con el progreso del abordaje laparoscópico, esté fue adquiriendo mayor rol protagónico.

Independientemente del tipo de abordaje la finalidad del drenaje es el mismo, consiste en comunicar la cavidad del pseudoquiste con el estómago o el intestino delgado permitiendo de esta manera disminuir la presión en su interior y favorecer su cicatrización.
El drenaje quirúrgico ha ido perdiendo terreno en mano de los tratamientos mínimamente invasivos como la endoscopia - el drenaje percutáneo, reservándose para aquellos casos que estos fracasaron. Dentro de los distintos procedimientos tenemos la cistogastroanastomosis, la cistoyeyunoanastomosis o la cistoduodenoanastomosis, que dependerá de la ubicación del pseudoquiste. La cistoyeyunoanastomosis en $Y$ de Roux se puede realizar para todos los pseudoquistes, por lo que es la técnica más frecuente a realizar. Según las series publicadas el drenaje quirúrgico tiene una mortalidad del 5-9\%, con un promedio de complicaciones alrededor del $11-24 \%$ y una recurrencia del $5-8 \%$.

Como se mencionó anteriormente el drenaje endoscópico se ha convertido en la primer opción terapéutica, presenta como ventaja que es un procedimiento mínimamente invasivo, tiene menor costo, menor estadía hospitalaria y ofrece mejor calidad de vida. Este puede ser transpapilar con colocación de stent o transmural. Este requiere la protrusión del pseudoquiste hacia la luz del órgano, el grosor de la pared quística inferior a $1 \mathrm{~cm}$ y la ausencia de grandes vasos. Para realizar un drenaje transpapilar debe existir comunicación entre el pseudoquiste y el conducto pancreático, para lo cual es de suma importancia realizar la colangioresonancia. Dentro de sus complicaciones tenemos sangrado, perforación intestinal, infección, migración del stent y recurrencia. Según los resultados publicados dicho procedimiento presenta una efectividad del $90-95 \%$, una tasa de complicaciones aproximadamente del $12 \%$, una recurrencia del $8 \%$ y una mortalidad del $1 \%{ }^{15}$.

El drenaje percutáneo puede realizarse bajo guía ecográfica, tomografía o bien radiológica. Esta técnica tiene éxito en la resolución del pseudoquiste pero en comparación con los otros métodos presenta mayor tasa de complicaciones por lo que recomiendan indicarlo en pacientes inestables. Dentro de sus complicaciones tenemos, fístulas, infecciones y obstrucción del catéter. Presenta una efectividad del $70-80 \%$, siendo mayor en intervencionistas experimentados, una tasa de complicaciones del $10-15 \%$ y una tasa de recidiva entre $15-20 \%$.

La resección quirúrgica es un procedimiento que presenta altas tasas de morbimortalidad, está indicado en duda diagnóstica de lesión quística tumoral, afectación trombótica de la vena esplénica, sangrado variceal que no respondió a embolización, pseudoquiste con conducto pancreático desconectado que fallaron otras terapéuticas y la incapacidad tecnológica para drenar pseudoquiste ubicado en el proceso uncinado ${ }^{16}$.

Dentro de las complicaciones del pseudoquiste tenemos; la hemorragia, la infección, por efecto de masa puede producir hipertensión portal y obstrucción de la vía biliar y ruptura que de producirse en el tubo digestivo no ocasionaría gravedad alguna en cambio de hacerlo en la cavidad abdominal puede desencadenar en sepsis $^{17}$. 


\section{Bibliografía}

1. Herrera Del Águila D, Garavito Rentería J, Linarez Medina , Lizarzaburu Rodríguez V. Pancreatitis aguda por hipertrigliceridemia severa: reporte de caso y revisión de la literatura. Rev. gastroenterol. Perú 2015; 35: 159-164.

2. Scherer J, Singh V, Pitchumoni C, Yadav D. Problemas en la pancreatitis hipertrigliceridémica: una actualización. J Clin Gastroenterol. 2014; 48: 195203

3. Jimenez Forero S, Roa Saavedra D, Villalba M. Pancreatitis aguda secundaria a hipertrigliceridemia: presentación de dos casos clínicos. Rev. Esp. Enferm. Dig. 2008; 100: 367-371.

4. Kyung Jeong $\mathrm{Y}, \mathrm{Kim} \mathrm{H}$. Una mini revisión sobre el efecto del ácido docosahexaenoico (DHA) en la pancreatitis aguda inducida por ceruleína e hipertrigliceridémica. Int J Mol Sci. 2017; 18: 2239.

5. Gutiérrez Restrepo J, Muñoz Ortiz E, Arango Toro C, Vásquez Mejía E, Montoya Escobar J, Villa Franco J. Pancreatitis aguda inducida por hipertrigliceridemia y tratamiento con plasmaféresis: reporte de un caso. latreia 2012; 25: 392-397.

6. Jimenez Forero S, Roa Saavedra D, Villalba M. Pancreatitis aguda secundaria a hipertrigliceridemia: presentación de dos casos clínicos. Rev. Esp. Enferm. Dig. 2008; 100: 367-371.

7. Gutiérrez Restrepo J, Muñoz Ortiz E, Arango Toro C, Vásquez Mejía E, Montoya Escobar J, Villa Franco J. Pancreatitis aguda inducida por hipertrigliceridemia y tratamiento con plasmaféresis: reporte de un caso. latreia 2012; 25: 392-397.

8. Jimenez Forero S, Roa Saavedra D, Villalba M. Pancreatitis aguda secundaria a hipertrigliceridemia: presentación de dos casos clínicos. Rev. Esp. Enferm. Dig. 2008; 100: 367-371.
9. Scherer J, Singh V, Pitchumoni C, Yadav D. Problemas en la pancreatitis hipertrigliceridémica: una actualización. J Clin Gastroenterol. 2014; 48: 195203.

10. Martínez Ordaz J, Toledo Toral C, Franco Guerrero N, Tun Abraham M, Souza Gallardo L. Surgical treatment of pancreatic pseudocysts. Rev. Cirugía y Cirujanos 2016; 84: 288-292.

11. Pan G, Hua W, Xie K, Li W, Hu W, Liu X. Clasificación y manejo de seudoquistes pancreáticos. Medicina Baltimore 2015; 94: 960.

12. Rodríguez D’Jesús A, Fernández Esparrach G, Saperas E. Tratamiento endoscópico del seudoquiste de páncreas: aspectos prácticos.G\&H. 2011; 34: 711-716.

13. Estepa Pérez J, Becerra G, Santana Pedraza T. Spontaneous Resolution for a Giant Pancreatic Pseudocyst. A Case Report. Medisur 2011; 9: 295-299.

14. Tenner S, Baillie J, DeWitt J, Vege S. Directriz del Colegio Americano de Gastroenterología: Manejo de la pancreatitis aguda. Am J Gastroenterol. 2013 108: $1400-1415$

15. Rodríguez D’Jesús A, Fernández Esparrach G, Saperas E. Tratamiento endoscópico del seudoquiste de páncreas: aspectos prácticos. G\&H. 2011; 34: 711-716.

16. Pan G, Hua W, Xie K, Li W, Hu W, Liu X. Clasificación y manejo de seudoquistes pancreáticos. Medicina Baltimore 2015; 94: 960.

17. Habashi S, Draganov P. Pseudoquiste pancreático. World J gastroenterol. 2009; 15: 38-47. 